\title{
UNIFIED MODELING OF MONOTONIC AND CYCLIC BEHAVIOR OF INTERFACE BETWEEN STRUCTURE AND GRAVELLY SOIL
}

\author{
GA ZHANG ${ }^{\text {i) }}$ and JIAN-MIN ZHANG ${ }^{\text {ii) }}$
}

\begin{abstract}
This paper describes an elasto-plasticity damage model, termed the EPDI model, of the interface between a structure and gravelly soil. The formulations are derived on the basis of a new model framework, with test-basis concepts and assumptions, for a unified description of monotonic and cyclic behavior of such an interface. The model parameters, with physical meaning, are easily determined from a group of cyclic shear tests and a confining compression test. The comparisons of model predictions with the results for a series of tests under varying loading conditions demonstrate that the EPDI model is effective in accurate description of monotonic and cyclic stress-strain relationship of the interface between a structure and gravelly soil with capturing new features, including: 1) shear stress-strain relationship and comprehensive volumetric strain response (e.g., partly dependent on shear strain); 2) volumetric strain response that is dependent on the shear direction; and 3) evolution of behavior of the interface associated with the evolution of physical state.
\end{abstract}

Key words: constitutive model, gravelly soil, interface, monotonic and cyclic behavior, soil-structure interaction (IGC: D6/E12/E13)

\section{INTRODUCTION}

Accurate modeling of the monotonic and/or cyclic behavior of a soil-structure interface is one of the key topics in the analysis of soil-structure systems such as high embankments, cut-off walls, earth reinforcements, deep foundations, and tunnels. Thus, a constitutive model of a soil-structure interface has become of great concern in recent years because of rapid developments in numerical methods and increasing requirements for such a model from those involved in the design of large-scale construction projects.

Constitutive models of a soil-structure interface are generally of four types: (1) Ideal models, such as an elasto-ideal plasticity model and a rigid plasticity model. These types of models are commonly referred to as "Mohr-Coulomb models" because the model's strength criterion is usually formulated using the Mohr-Coulomb criterion. For example, Brandt (1985) proposed a rigid plasticity model of an interface according to direct test results. (2) Nonlinear elasticity models. Clough and Duncan (1971) presented a model of an interface assuming a hyperbolic tangential stress-displacement relationship under a constant normal stress condition. Desai et al. (1985) modified the Romberg-Osgood model to describe the cyclic tangential stress-displacement relationship of an interface. These models are widely used in numerical analy- sis because of their simplicity, however, they cannot adequately simulate the plastic deformation and volumetric changes of a soil-structure interface. (3) Elasto-plasticity models. Ghaboussi et al. (1973) proposed one of the first elasto-plasticity interface models using a cap yield surface. A series of elasto-plasticity interface models have been developed on the basis of various assumptions (e.g., Fisherman and Desai, 1987; Fisherman et al., 1991; Gens, 1990; Boulon, 1990; Navayogarajah et al., 1992). (4) Damage models. The concept "damage" is used to describe the change in behavior of the interface with a diverse range of modifications. For example, Desai and Ma (1992) proposed a new "Disturbed State Concept" (DSC) and described a DSC model of the interface. A number of damage models that make use of the DSC or other damage concepts have been developed and used to predict the behavior of a soil-structure interface (e.g., Navayogarajah et al., 1992; Pal and Wathugala, 1999; Fakharian and Evgin, 2000; $\mathrm{Hu}$ and $\mathrm{Pu}, 2004)$. It can be found from the literature that an effective model is dependent strongly on the understanding of the behavior and deformation mechanism of a soil-structure interface.

Gravelly soil has become a great concern in the design and construction of large-scale projects such as high rockfill embankments, deep cut-off walls, and high-speed railways. For example, many rockfill embankments greater than $100 \mathrm{~m}$ in height are under construction in

i) Ph. D, Associate Professor, State Key Laboratory of Hydroscience and Engineering, Tsinghua University, Beijing, P R China (zhangga@ tsinghua.edu.cn).

ii) Professor, ditto.

The manuscript for this paper was received for review on July 26, 2007; approved on December 31, 2007.

Written discussions on this paper should be submitted before November 1, 2008 to the Japanese Geotechnical Society, 4-38-2, Sengoku, Bunkyo-ku, Tokyo 112-0011, Japan. Upon request the closing date may be extended one month. 
China. In a previous study, we conducted a large number of serialized tests on the monotonic and cyclic behavior of interfaces between a structure and gravelly soil using a large-scale test apparatus and image measurement techniques (Zhang and Zhang, 2006a; Zhang et al., 2006). We obtained the systematic results of the interface under different loading conditions, including both the macroscopic response of the stress-displacement relationship and microscopic changes in the deformation and the physical state (e.g., Zhang and Zhang, 2006b). Behavior of the interface between a structure and gravelly soil was found from the test results with some new features, including significant change of the physical state, asymmetrical response in the stress-displacement relationship (especially in the volumetric change) due to symmetrical loading in different directions, and comprehensive deformation mechanism, e.g., the volumetric change due to dilatancy is dependent on only a part of tangential displacement. These features are not adequately captured by the existing models that are usually designed for the interface between a structure and sand or clay. A reasonable model for such an interface may require a comprehensive understanding of the macroscopic stress-displacement relationship and microscopic deformation mechanism, which is derived from the test results.

The objective of this paper is to establish an effective constitutive model, based on the solid test-based evidences, for a unified description of monotonic and cyclic behavior of the interface between a structure and gravelly soil, including: (1) to present necessary concepts and assumptions along with experimental and theoretical proofs; (2) to propose a model framework; (3) to obtain mathematical formulations; (4) to discuss model parameters and how they are determined; and (5) to confirm the effectiveness of the model by comparison of model predictions with test results.

\section{FRAMEWORK}

\section{Assumptions}

Constant Strain Assumption

The interface has thickness, which is invariable during loading. The strain of the interface is constant.

This assumption is based on the results of micro-observations of the tests. For example, Fig. 1 shows horizontal translations, relative to the soil container, of soil particles near the structure in a monotonic shear test of an interface. Here the displacement of the soil container is equal to the tangential displacement of the interface. Significant particle movements occur in a narrow zone extending from the structural surface. This demonstrates that the interface has a thickness. The tangential displacement has only a small effect on the distance from the structure at which the movements become negligible although they significantly affect the movement of soil particles (Fig. 1); so does the normal stress (Zhang and Zhang, 2006b). We concluded that the thickness of the interface between a structure and gravelly soil can be considered to be constant during loading. This thickness can

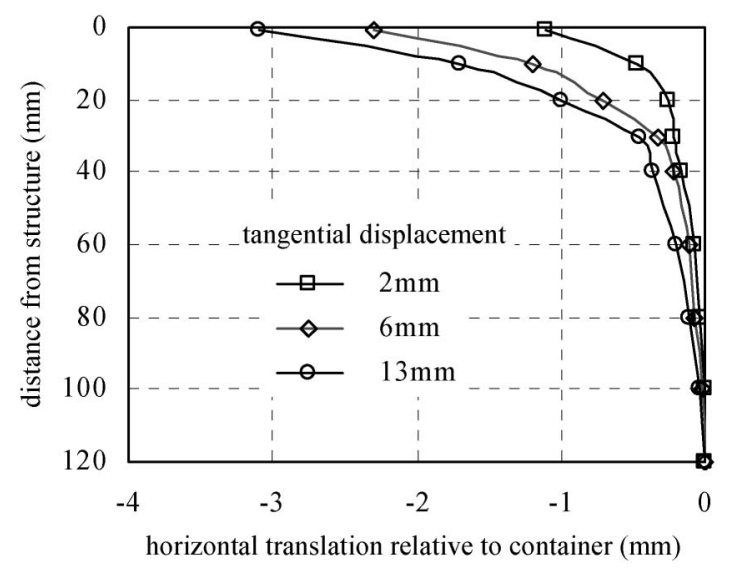

Fig. 1. Movements of soil particles near the structure in a monotonic shear test of steel-gravel interface under constant normal stress of $200 \mathrm{kPa}$, measured using image analysis. Translation is defined as positive if it occurs in the same direction as that of the soil container

be determined by measuring the movements of soil particles. For example, the thickness of this interface is approximately taken five- to six-times the average grain-size of the soil; it is consistent with the knowledge of the thickness of the interface between a structure and sand (e.g., Uesugi and Kishida, 1986).

Although a nonlinear distribution was found within the interface (Fig. 1), the strain of the interface is assumed to be a constant for simplicity. Thus, using the thickness of the interface, $t$, the shear strain, $\gamma$, and the volumetric strain, $\varepsilon_{\mathrm{v}}$, can be determined from the tangential displacement, $u$, and normal displacement, $v$, of the interface. That is,

$$
\left\{\begin{array}{c}
\gamma=\frac{u}{t} \\
\varepsilon_{\mathrm{v}}=\frac{v}{t}
\end{array}\right.
$$

Non-elasticity Assumption

Elastic and plastic strains are always simultaneously induced by stress.

This assumption is based on an understanding of the behavior of geomaterials subjected to a complex stress path. Thus, strain can be written in the following increment form:

$$
\left\{\begin{array}{l}
d \gamma=d \gamma^{\mathrm{e}}+d \gamma^{\mathrm{p}} \\
d \varepsilon_{\mathrm{v}}=d \varepsilon_{\mathrm{v}}^{\mathrm{e}}+d \varepsilon_{\mathrm{v}}^{\mathrm{p}}
\end{array}\right.
$$

where $\gamma^{\mathrm{e}}$ and $\gamma^{\mathrm{p}}$ are the elastic and plastic shear strain, respectively; and $\varepsilon_{\mathrm{v}}^{\mathrm{e}}$ and $\varepsilon_{\mathrm{v}}^{\mathrm{p}}$ are the elastic and plastic volumetric strain, respectively. The symbol ' $d$ ' represents the increment of a strain.

\section{Uncoupling Elastic Strain Assumption}

The components of elastic strain are only dependent on the corresponding stress component.

This assumption indicates that elastic shear strain is only induced by shear stress, while elastic volumetric 
strain is only induced by normal stress.

Decomposition of Plastic Strain Assumption

The plastic strain is divided into two components: one due to shear and the other due to compression. The plastic strain component due to shear includes the shear strain and volumetric strain components. The plastic strain component due to compression consists solely of the volumetric strain component, which is induced only if the normal stress is greater than the maximum historical normal stress that the interface has undergone.

This assumption can simplify the modeling while preserving physical rationality. It is suitable for a soilstructure interface because the behavior due to shear can be considered. Using this assumption, the plastic volumetric strain can be written in increment form as follows:

$$
d \varepsilon_{\mathrm{v}}^{\mathrm{p}}=d \varepsilon_{\mathrm{vc}}^{\mathrm{p}}+d \varepsilon_{\mathrm{vd}}
$$

where $\varepsilon_{\mathrm{vd}}$ and $\varepsilon_{\mathrm{vc}}^{\mathrm{p}}$ are the volumetric strain due to shear and plastic volumetric strain due to compression, respectively. The symbol ' $d$ ' represents the increment of a strain.

\section{Concepts}

Reversible and Irreversible Volumetric Strain due to Dilatancy

Under a constant normal stress condition, the volumetric strain, which is derived from normal displacement using Eq. (1), is induced solely by changes in shear stress or shear strain because the normal stress and interface size is maintained over the entire shearing test. Such a volumetric strain induced by shear application is termed as "volumetric strain due to dilatancy" and denoted by $\varepsilon_{\mathrm{vd}}$. $\varepsilon_{\mathrm{vd}}$ gradually accumulates and fluctuates in magnitude with the repeated increases and decreases in shear stress during the application of cyclic shear (Fig. 2). The maximum values of $\varepsilon_{\mathrm{vd}}$ attained during each shear cycle are plotted in Fig. 2 using a broken curve. This curve shows an irreversible, gradually increasing component of volumetric strain due to dilatancy that is mainly determined by the shear history. In addition, Fig. 2 also shows the result of subtracting the irreversible component from $\varepsilon_{\mathrm{vd}}, \varepsilon_{\mathrm{vd} \text {, re }}$. The new component can be found recoverable over the shear process.

Thus, $\varepsilon_{\mathrm{vd}}$ can be divided into two types of components: one characterized by its reversibility and dependency on the magnitude and direction of the current shear strain, and another characterized by its irreversibility and dependency on the shear history. As a result, the former is referred to as "reversible volumetric strain due to dilatancy", and denoted as $\varepsilon_{\mathrm{vd}, \text { re }}$, while the latter is termed "irreversible volumetric strain due to dilatancy" and denoted as $\varepsilon_{\mathrm{vd}, \text { ir }}$. In general, this can be expressed as:

$$
\varepsilon_{\mathrm{vd}}=\varepsilon_{\mathrm{vd}, \text { ir }}+\varepsilon_{\mathrm{vd}, \text { re }}
$$

The observed changes in the reversible dilatancy component of the interface within different shear cycles show that $\varepsilon_{\mathrm{vd}, \text { re }}$ within a single shear cycle is nearly closed, which again indicates that it is "reversible" (Fig. 3). $\varepsilon_{\mathrm{vd} \text {, re }}$
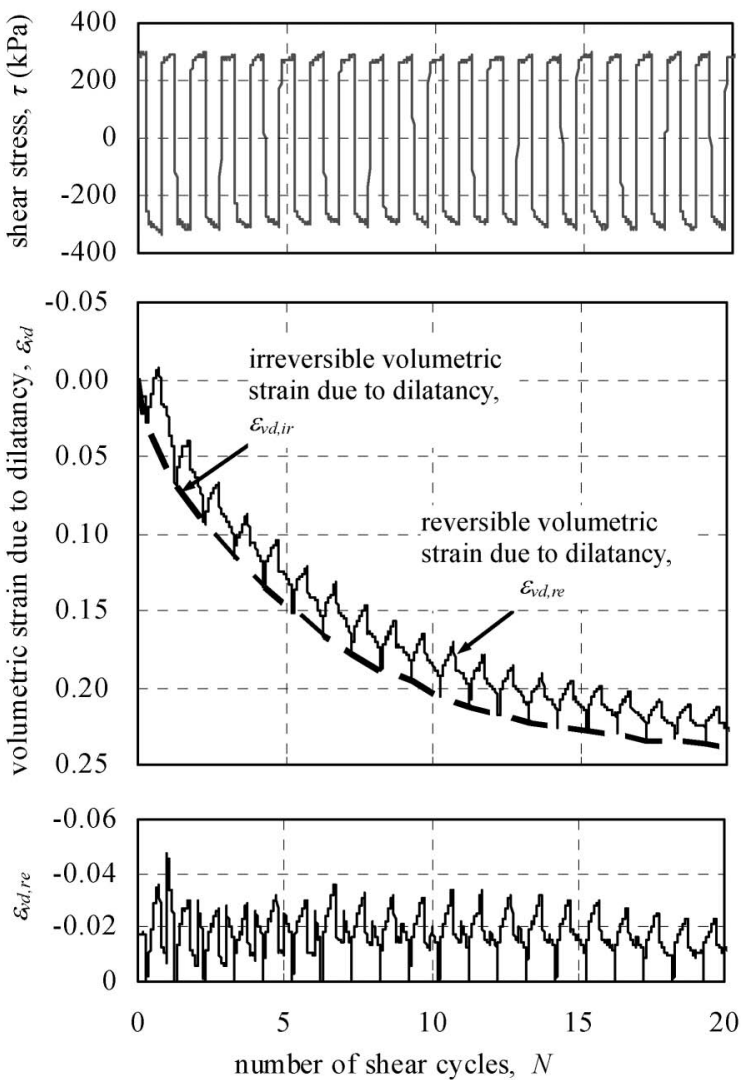

Fig. 2. Reversible and irreversible volumetric strain due to dilatancy of the cyclic shear test of steel-gravel interface under constant normal stress of $400 \mathrm{kPa}$ (thickness of the interface: $50 \mathrm{~mm}$ )

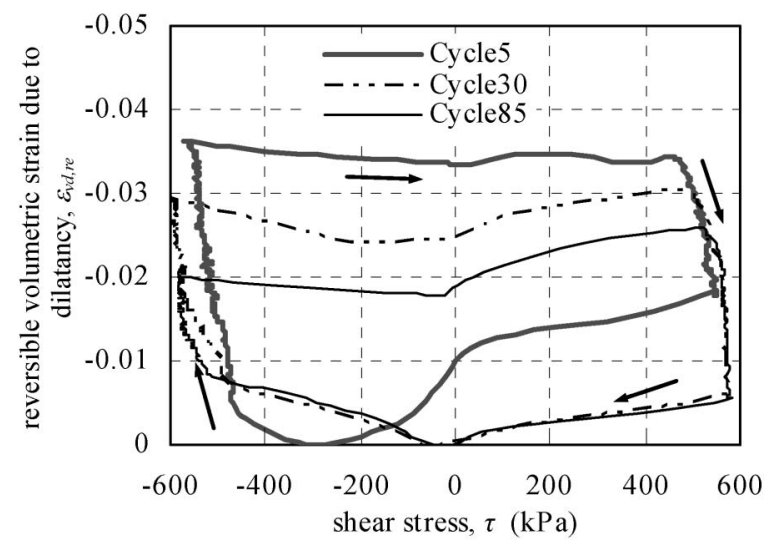

Fig. 3. Reversible volumetric strain due to dilatancy of steel-gravel interface due to cyclic shear application under constant normal stress of $700 \mathrm{kPa}$ (thickness of the interface: $50 \mathrm{~mm}$ ) arrow: shear direction

is dependent on the magnitude and direction of shear strain or shear stress. Its changes within a single shear cycle between different shear cycles become progressively smaller with increasing number of shear cycles. $\varepsilon_{\mathrm{vd}}$, re $\mathrm{ex}-$ hibits a significant behavior that its change trend is dependent on the shear direction. The transition point of shear stress, at which the change tendency of $\varepsilon_{\mathrm{vd}}$, re turns from compressing to dilating, moves to zero during shearing from a certain value at the beginning of shear. 


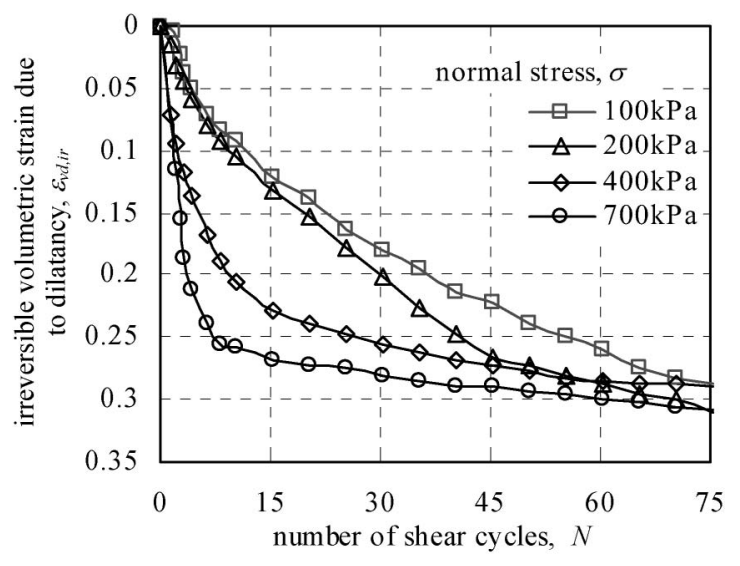

Fig. 4. Irreversible volumetric strain due to dilatancy of steel-gravel interface due to cyclic shear application under constant normal stress conditions (thickness of the interface: $50 \mathrm{~mm}$ )

The test results also indicate that the amplitude of $\varepsilon_{\mathrm{vd} \text {, re }}$ generally decreases with increasing normal stress.

The irreversible volumetric strain due to dilatancy, $\varepsilon_{\mathrm{vd}, \text { ir }}$, gradually increases at a decreasing rate of change before finally achieving a stable value with increasing number of shear cycles (Fig. 4). The normal stress has an insignificant effect on the stability value of $\varepsilon_{\mathrm{vd}}$, ir , but has a positive influence on the rate of change during the first few shear cycles.

\section{Aeolotropy of Interface}

The cyclic test results of the interface between a structure and gravelly soil show that the mechanical response, e.g., shear strength and volumetric strain due to dilatancy, is dependent on shear direction after an initial shear application (e.g., Zhang and Zhang, 2006b). This new behavior that mechanical response is dependent on the shear direction is defined as "aeolotropy of interface". It is different from the traditional concept of "anisotropy", because the asymmetric response is induced in different directions on a line, for example, in a two-way cyclic shear test. Another concept, homotropy of interface, is correspondingly used to describe the behavior in which mechanical response is independent of the shear direction. A main reason that the aeolotropy of interface comes into being is probably the initial shear application, as this brings about structural aeolotropy of the arrangements and dip directions of the soil particles near the structure because of the constraint of the structure. The initial shear history is found to be a main factor influencing the extent of aeolotropy of interface (Zhang and Zhang, 2006b).

The test results indicate that the reversible volumetric strain due to dilatancy, $\varepsilon_{\mathrm{vd}}$, re, exhibits significant aeolotropy of interface. For example, it mainly increases when shear stress is applied in the initial shear application, whereas it mainly decreases in the reverse direction (Fig. 3). This change tendency becomes more significant after several shear cycles. To simplify the formulation, in this paper, $\varepsilon_{\mathrm{vd} \text {, re }}$ is divided into a homotropic reversible dilatancy component, $\varepsilon_{\mathrm{vd}, \mathrm{re}, \mathrm{h}}$, and an aeolotropic reversible dilatancy component, $\varepsilon_{\mathrm{vd}, \text { re, a }}$. That is,

$$
\varepsilon_{\mathrm{vd}, \mathrm{re}}=\varepsilon_{\mathrm{vd}, \mathrm{re}, \mathrm{h}}+\varepsilon_{\mathrm{vd}, \mathrm{re}, \mathrm{a}}
$$

\section{Effective Shear Strain}

The measurement results of the soil particle movements show that the horizontal translations, relative to the soil container are always smaller than the tangential displacement of the soil container (Fig. 1); this finding has been confirmed by the microscopic measurements of many tests (Zhang and Zhang, 2006b). This demonstrates that tangential displacement, i.e., shear strain, of the interface comprises a slippage component at the contact face, and a soil deformation component constrained by the structure. The two components occur at the same time, but their proportion varies according to the magnitude of tangential displacement (Fig. 1). We have concluded that deformation of the soil constrained by the structure is the main contributor to volumetric change due to dilatancy (Zhang and Zhang, 2006b). Thus, it is reasonable to establish the relationship between volumetric strain due to dilatancy and shear strain due to deformation of the soil constrained by the structure.

Accordingly, a new concept, "effective shear strain", is defined to help us consider the shear strain upon which the volumetric strain due to dilatancy is dependent. The "effective shear strain" refers to the component of shear strain that contributes to the volumetric strain due to dilatancy. This term is equivalent to the shear strain induced by the deformation of soil within the interface in light of the deformation mechanism. It can be formulated as a function of the shear strain and normal stress.

\section{Evolution of Physical State}

Close examinations of the photographs of the structure and nearby soil over the shearing process show that crushing of soil particles occurs near the structure from the moment that shear is first applied and becomes significant after several shear cycles. A definition termed "thickness of crushing band", $D_{\text {c }}$, has been used to provide an approximate description of the crushing extent of soil particles during the shear test (Zhang and Zhang, 2006b). $D_{\text {c }}$ can be estimated as the thickness of the zone within which significant crushing of soil particles can be observed from the photographs. With the application of cyclic shear under constant normal stress condition, the thickness of crushing band monotonically increases to a stable value with increasing number of shear cycles (Fig. 5). This demonstrates that the particle-crushing of the soil near the structure reaches a stable state with continued application of shear. It has been indicated that the irreversible volumetric strain due to dilatancy also gradually increases with increasing number of shear cycles (Figs. 4-5); this demonstrates that the soil near the structure is continuously compressed due to cyclic shear application. Thus, we conclude that there is a significant evolution in the physical state, including particle-crushing and compression of the soil near the structure due to shear application. 


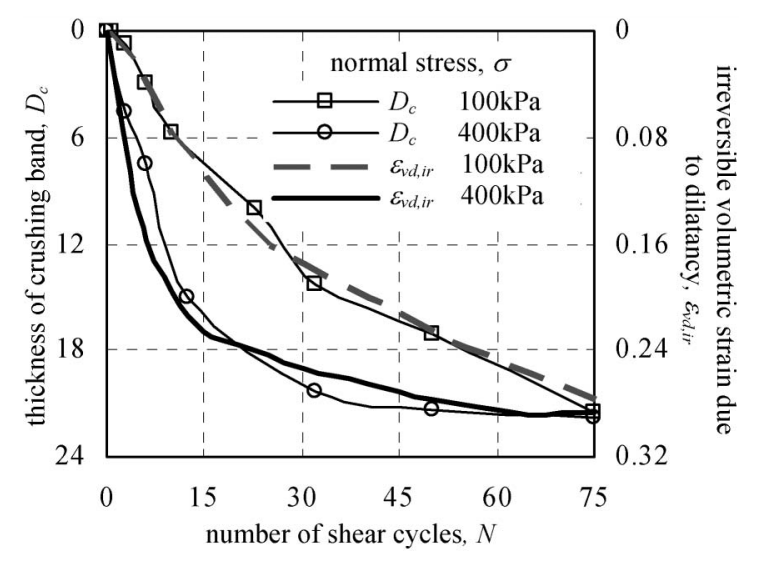

Fig. 5. Thickness of crushing band and irreversible volumetric strain due to dilatancy in the shear test of steel-gravel interface under constant normal stress condition (thickness of the interface: $50 \mathrm{~mm}$ )

It is evident from Fig. 5 that $\varepsilon_{\mathrm{vd}}$, ir and $D_{\mathrm{c}}$ show fairly consistent patterns of development; this demonstrates that the crushing of soil particles is an important factor in the irreversible volumetric strain due to dilatancy. In other words, the particle-crushing and compression of soil near the structure have a close relation.

The evolution of the physical state results in the evolution of the behavior of the interface during shear application; thus the macroscopic stress-displacement relationship evolves from the initial state to a stable state. This finding can be demonstrated from test results related to the fact that the stress-displacement relationship always develops with a tendency to a stable state during shear application, including that: 1) the loading curve of the tangential stress-displacement relationship exhibits a tendency to linearity, as with the unloading curve (Zhang and Zhang, 2006b); 2) $\varepsilon_{\mathrm{vd}}$, ir increases gradually at a decreasing rate toward a stable value (Fig. 4); and 3) $\varepsilon_{\mathrm{vd} \text {, re }}$ records an evolution in both the amplitude and transition point of the shear stress (Fig. 3).

To describe the evolution of the physical state and the resulting evolution of behavior of the interface, the damage model frame is used in this paper. The traditional damage concept (Kachanov, 1958) had been extended, with a few important modifications, to describe the behavior of geomaterials. For example, a material at a critical state can bear spherical stress (Frantziskonis and Desai, 1987) and even shear stress (Shen, 1988). In this paper, the "damage" concept is extended to characterize the evolution of the microscopic physical state and the resulting evolution of the macroscopic behavior. The concepts of "initial state", "ultimate state", and "damage variable" are accordingly defined. The "initial state" is defined as the original state at which the physical state has yet to be disturbed by loading. The "ultimate state" is defined as the stable state at which the physical state is invariable; thus most model parameters at the ultimate state can be constants. It should be noted that the "ultimate state" is distinct from the traditional concept of "critical state" by virtue of the specification that shear strength and other characteristics are stable, but not lost, at the ultimate state. The "damage variable", $D$, is used to measure the evolution extent of the physical state and behavior from the initial state to the ultimate state.

It is assumed that some behavior parameters at an intermediate state can be determined by using the corresponding parameters at the initial and ultimate states, weighted by the damage variable. In other words, a behavior parameter at an arbitrary state, $H$, can be determined as:

$$
H=(1-D) \cdot H_{0}+D \cdot H_{\mathrm{u}}
$$

where $H_{0}$ and $H_{\mathrm{u}}$ are the behavior parameter at the initial and ultimate states, respectively. In this paper, the subscript " 0 "' represents the initial state and ' $u$ "' represents the ultimate state.

It was demonstrated that the irreversible volumetric strain due to dilatancy always monotonically increases from zero at the initial state to its maximum value, $\varepsilon_{\mathrm{vd} \text {, ir, ult }}$, at the ultimate state. Thus, the damage variable is determined using the normalization of $\varepsilon_{\mathrm{vd}}$, ir, i.e.,

$$
D=\frac{\varepsilon_{\mathrm{vd}, \text { ir }}}{\varepsilon_{\mathrm{vd}, \text { ir, ult }}}
$$

Obviously, $D$ ranges from 0 at the initial state to 1 at the ultimate state. A number of statements in support of Eq. (7) can be presented on the basis of the behavior and deformation mechanism of the interface between a structure and gravelly soil, including: (1) Damage (i.e., evolution of physical state) of the interface is induced mainly by the application of shear; and $\varepsilon_{\mathrm{vd}}$, ir is dependent on the shear history. (2) The main reason for the damage is particle-crushing and compression of the soil near the structure due to shear application; this results in an irreversible volumetric change. This volumetric change is exactly the irreversible volumetric strain due to dilatancy if the

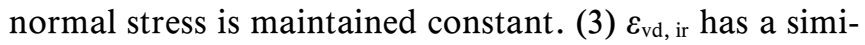
lar development tendency to that of the stress-displacement relationship under an applied shear. Its development feature is also qualitatively consistent with that of the particle-crushing extent (Fig. 5). Thus, it can be used as a measure of the evolution extent of the physical state and that of the behavior of the interface. (4) $\varepsilon_{\mathrm{vd}}$, ir has a definitive physical meaning and is easily measured.

Thus, the damage's law is presented based on a mechanism analysis that combines the results of macroand micro-observations, and artificial assumption is avoided.

\section{Framework}

On the basis of the above assumptions and concepts, a new model framework is proposed by introducing "bounding surface" (Fig. 6). The bounding surface model is recognized for its reasonable and concise description of the cyclic behavior of a geomaterial, for example, the reversal of a stress path. Mroz (1967) and Dafalias and Herrmann (1980) respectively proposed bounding surface models for soil.

Based on the extended "damage" concept, the evolution of behavior can be formulated using the behavior at 


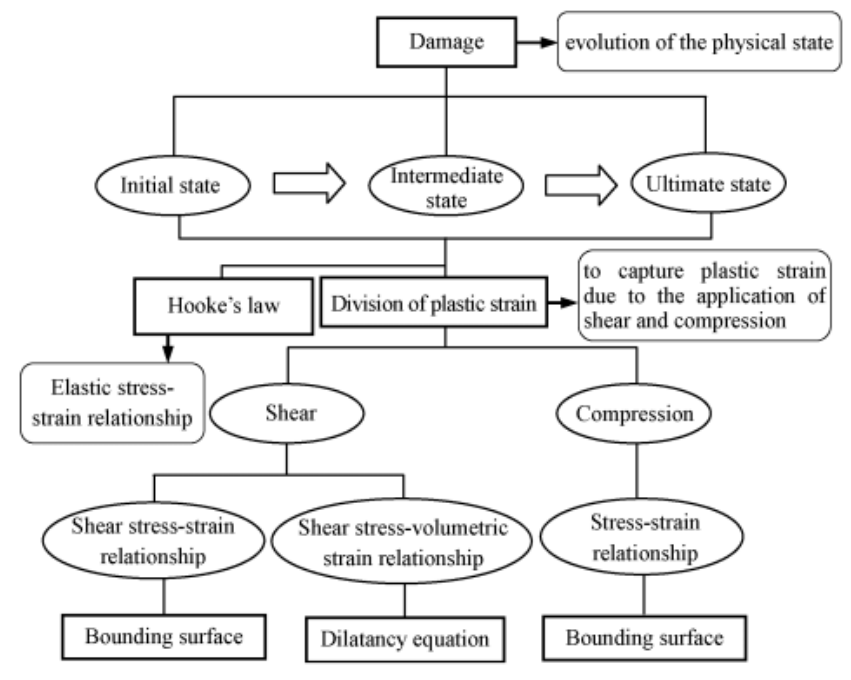

Fig. 6. Framework of the EPDI model

the initial and ultimate states according to the damage's law. The behaviors at the initial and ultimate states are both formulated using the same form so that the evolution of behavior is simplified to the evolution of a few behavior parameters. The "Decomposition of plastic strain" assumption means that plastic strain is divided into two components: one is due to shear, and the other is due to compression. Both components are respectively formulated based on bounding surface concepts, which describe the turning of a stress path by changing the mapping point and compute the deformation modulus according to the mapping length. A dilatancy equation is directly proposed to compute the volumetric strain due to dilatancy, using several important concepts such as reversible/irreversible volumetric strain due to dilatancy and effective shear strain. The non-elasticity assumption provides a theoretical basis for the dilatancy equation of the reversible dilatancy component.

The model is based on this framework, which in turn is based on elasto-plasticity and damage. Thus, the model is referred to as the "Elasto-Plasticity Damage model of the Interface between a structure and gravelly soil" (EPDI model).

\section{FORMULATIONS}

According to the assumptions and concepts, Eqs. (2)-(5), shear strain, $\gamma$, and volumetric strain, $\varepsilon_{v}$, can be expressed in an increment form as follows:

$$
\left\{\begin{array}{l}
d \gamma=d \gamma^{\mathrm{e}}+d \gamma^{\mathrm{p}} \\
d \varepsilon_{\mathrm{v}}=d \varepsilon_{\mathrm{vc}}^{\mathrm{e}}+d \varepsilon_{\mathrm{vc}}^{\mathrm{p}}+d \varepsilon_{\mathrm{vd}, \mathrm{re}, \mathrm{h}}+d \varepsilon_{\mathrm{vd}, \mathrm{re}, \mathrm{a}}+d \varepsilon_{\mathrm{vd}, \text { ir }}
\end{array}\right.
$$

where $\varepsilon_{\mathrm{vc}}^{\mathrm{e}}$ is the elastic volumetric strain due to compression. The mathematical formula for each component is presented at the initial and ultimate states, respectively. Thus, the expression at an arbitrary state can be derived from the damage's law, Eqs. (6)-(7).

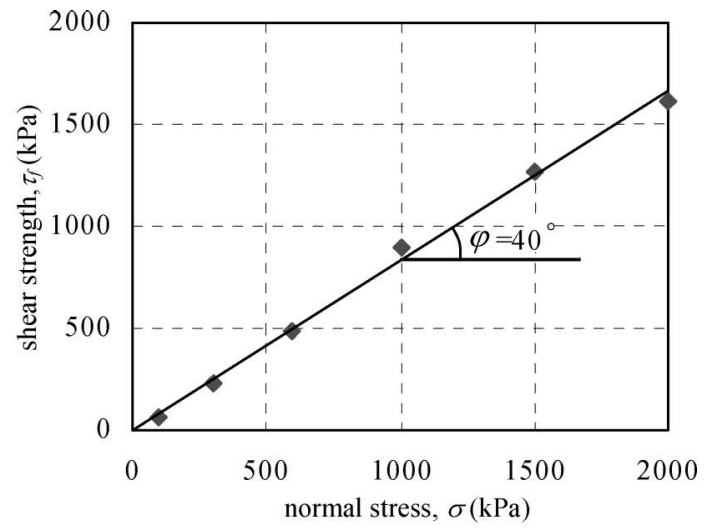

(a)

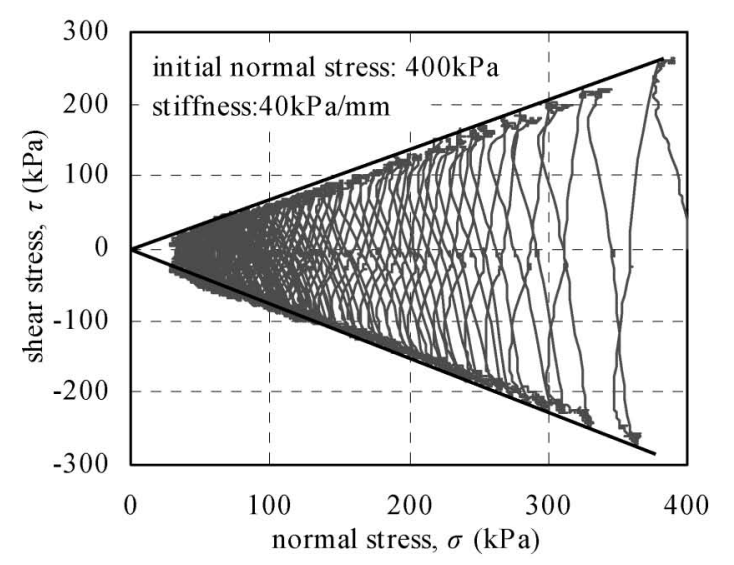

(b)

Fig. 7. Shear strength of interface between a structure and gravelly soil: (a) monotonic relationship between shear strength and normal stress of the concrete-gravel interface of Gongboxia CFRD under constant normal stress condition and (b) stress path of a steel-gravel interface under a constant normal stiffness boundary $\varphi$ : friction angle; symbol: test result; line: linear fit

\section{Shear Strength}

We conclude from the test results that the shear strength is proportional to normal stress (e.g., Fig. 7(a)); and this is confirmed by the straight envelope line of the stress path obtained from cyclic shear test under a constant normal stiffness condition (e.g., Fig. 7(b)). Thus, shear strength, $\tau_{\mathrm{f}}$, can be formulated solely from the friction angle, $\varphi$, i.e.,

$$
\tau_{\mathrm{f}}=\sigma \tan \varphi
$$

where $\varphi$ is a model parameter.

\section{Shear Strain}

The elastic strain at an arbitrary state is formulated as follows:

$$
d \gamma^{\mathrm{e}}=\frac{d \tau}{G_{\mathrm{e}}}
$$

where the elastic shear modulus, $G_{\mathrm{e}}$, can be set to a constant of $10^{5} \mathrm{kPa}$.

A bounding surface scheme is described to compute the 


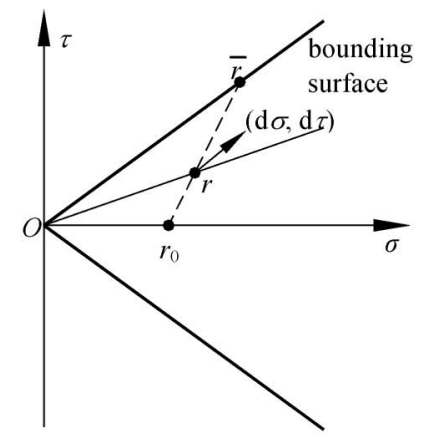

(a)

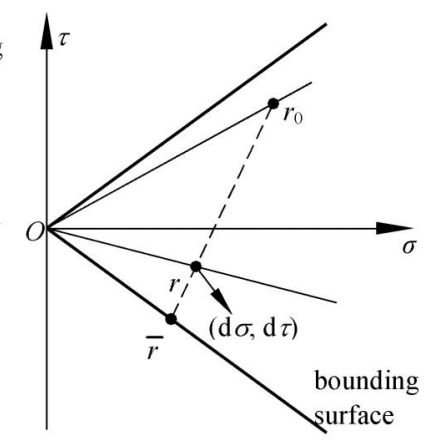

(b)
Fig. 8. Bounding surface scheme: (a) initial application of shear and (b) application of cyclic shear

plastic shear strain due to shear application (Fig. 8). This scheme consists of a bounding surface and three important stress points: a mapping point, $r_{0}$; a stress point, $r$; and a conjugate point, $\bar{r}$. The conjugate point is the crossing point of the bounding surface and the extension line from the mapping point to the stress point (Fig. 8).

$\rho_{0}$ is defined as the distance from the mapping point to the conjugate point, and $\rho$ is defined as the distance from the mapping point to the stress point. That is,

$$
\rho_{0}=\left|r_{0} \bar{r}\right| ; \quad \rho=\left|r_{0} r\right|
$$

Clearly, $\rho_{0} \geq \rho$. The ratio of $\rho$ to $\rho_{0}$ is defined as an internal variable of the stress state. For example, if $\rho / \rho_{0}=0$, the shear modulus is solely the initial one. In contrast, if $\rho / \rho_{0}=1$, the interface has reached a 'flow' state and its shear modulus is zero. It is possible that the conjugate point cannot be found because the bounding surface is not closed. In such a case, $\rho_{0}$ is considered to be infinite, and thus we define $\rho / \rho_{0}=0$.

The bounding surface is determined by the shear strength, $\tau_{\mathrm{f}}$. That is,

$$
\boldsymbol{F}=\tau^{2}-\tau_{\mathrm{f}}^{2}=0
$$

Loading is judged using the following equation:

$$
d f_{\mathrm{s}}=\sigma \cdot d|\tau|-|\tau| \cdot d \sigma \quad\left\{\begin{array}{l}
d f_{\mathrm{s}} \geq 0: \text { Loading } \\
d f_{\mathrm{s}}<0: \text { Unloading }
\end{array}\right.
$$

The mapping point, $r_{0}$, is obtained according to the following rules: (1) Under the initial loading condition (i.e., monotonic loading), the mapping point is the initial loading point at which the first shear stress increment is applied (Fig. 8(a)). (2) Under the cyclic loading condition, there are two possible cases: I) if the stress state converts from loading to unloading due to the stress increment, the mapping point is just the transition point (Fig. 8(b)); II) if the stress state converts from unloading to loading, an additional definition is needed to determine the mapping point. The transition point can be used as the mapping point only if the angle between the current shear stress increment, $d \tau$, and the previous shear increment, $d \tilde{\tau}$, is greater than $90^{\circ}$; that is, satisfying

$$
d \tau \cdot d \tilde{\tau}<0
$$

The test results show that the shear stress-strain relationship can be formulated using a hyperbola under a constant normal stress condition (Zhang and Zhang, 2006b). This relationship is substituted as an extreme case into the bounding surface scheme. To consider the effect of normal stress, assume that the initial shear modulus is the exponential function of the normal stress at the initial state, whereas it is linear to the normal stress at the ultimate state. Therefore, the plastic shear strains are derived at the initial and ultimate states, respectively. Thus, the plastic shear strain at an arbitrary state is finally derived using Eq. (6), that is,

$$
d \gamma^{\mathrm{p}}=\frac{1}{H_{\mathrm{r}}} d \tau-\frac{1}{H_{\mathrm{rd}}} \frac{|\tau|}{\sigma} d \sigma
$$

where

$$
\begin{aligned}
& H_{\mathrm{rd}}=(1-D) G_{0} p_{\mathrm{a}}\left(\frac{\sigma}{p_{\mathrm{a}}}\right)^{\mathrm{n}_{0}}+D \cdot G_{\mathrm{c}} \sigma, \\
& H_{\mathrm{r}}=\left(1-\frac{\rho}{\rho_{0}}\right)^{2} H_{\mathrm{rd}}
\end{aligned}
$$

where the initial shear modulus, $G_{0}$, and shear index, $n_{0}$, are model parameters; the ultimate shear modulus, $G_{\mathrm{u}}$, can be set to $2 G_{0}$ in this paper. $p_{\text {a }}$ is the standard atmosphere (101325 Pa).

\section{Irreversible Volumetric Strain due to Dilatancy}

$\varepsilon_{\mathrm{vd}}$, ir has an extreme value, and normal stress is a principle factor influencing the initial increase rate of this strain. Thus, the relationship between the effective shear strain and $\varepsilon_{\mathrm{vd}}$, ir is formulated using a hyperbola under a constant normal stress condition. By considering the effect of normal stress, $\varepsilon_{\mathrm{vd} \text {, ir }}$ can be expressed as:

$$
\varepsilon_{\mathrm{vd}, \text { ir }}=\frac{\bar{\gamma}}{\frac{\bar{\gamma}}{\varepsilon_{\mathrm{vd}, \text { ir, ult }}}+\alpha\left(\frac{\sigma}{p_{\mathrm{a}}}\right)^{-\beta}}
$$

where the irreversible dilatancy modulus, $\alpha$, the irreversible dilatancy index, $\beta$, and $\varepsilon_{\mathrm{vd}}$, ir, ult are model parameters. $\bar{\gamma}$ is the effective shear strain corresponding to the irreversible volumetric strain due to dilatancy, which is the sum of the effective shear strain increments if the increment form is employed.

A comparison of Eq. (17) in terms of model predictions and test results for an interface due to cyclic shear application shows that the predictions are in good agreement with the test results (Fig. 9); this confirms the effectiveness of such an equation. By finding the derivation of Eq. (17) and making appropriate modifications to ensure that $d \varepsilon_{\mathrm{vd}}$, ir increases monotonically even if normal stress decreases, the increment equation for $\varepsilon_{\mathrm{vd}}$, ir is derived as:

$$
d \varepsilon_{\mathrm{vd}, \text { ir }}=\left(\frac{1}{B}-\frac{\bar{\gamma}_{\mathrm{mob}}}{B^{2} \varepsilon_{\mathrm{vd}, \text { ir, ult }}}\right)|d \bar{\gamma}|
$$

where the symbol ' $d$ ' represents the increment of the strain; and 


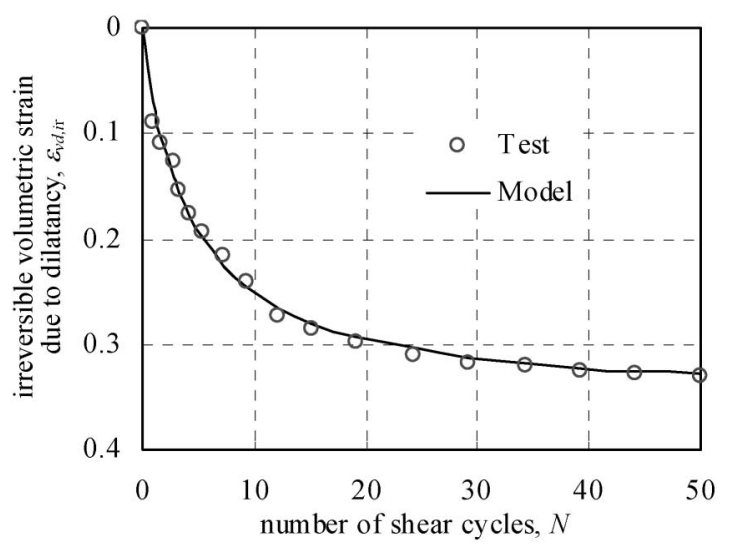

Fig. 9. Model predictions and test results for irreversible volumetric strain due to dilatancy of steel-gravel interface under constant normal stress of $400 \mathrm{kPa}$

$$
\bar{\gamma}_{\mathrm{mob}}=\alpha\left(\frac{\sigma}{p_{\mathrm{a}}}\right)^{-\beta} \frac{\varepsilon_{\mathrm{vd}, \text { ir }}}{1-\frac{\varepsilon_{\mathrm{vd}, \text { ir }}}{\varepsilon_{\mathrm{vd}, \text { ir, ult }}}} \quad B=\frac{\bar{\gamma}_{\mathrm{mob}}}{\varepsilon_{\mathrm{vd}, \text { ir, ult }}}+\alpha\left(\frac{\sigma}{p_{\mathrm{a}}}\right)^{-\beta}
$$

The effective shear strain component, $\bar{\gamma}$, is required to calculate $\varepsilon_{\mathrm{vd} \text {, ir }}$ according to an arbitrary shear strain. In this paper, we propose a hyperbola relationship between this effective shear strain and the plastic shear strain. Thus, the increment form is derived as:

$$
d \bar{\gamma}=\frac{b}{\left(\frac{\gamma_{1}^{\mathrm{p}}}{\bar{\gamma}_{\max }}+b\right)^{2}} d \gamma^{\mathrm{p}}
$$

where $\gamma_{1}^{\mathrm{p}}$ is the gross plastic shear strain accumulated from the beginning of a monotonic stress path. $\bar{\gamma}_{\max }$ and $b$ can be set to constants of 1 and 0.2 , respectively.

Considering the formulation of plastic shear strain provided in Eq. (15), $\varepsilon_{\mathrm{vd}, \text { ir }}$ is expressed in the stress increment form as follows:

$$
d \varepsilon_{\mathrm{vd}, \mathrm{ir}}=A_{1}\left(\frac{1}{H_{\mathrm{r}}} d|\tau|-\frac{1}{H_{\mathrm{rd}}} \frac{|\tau|}{\sigma} d \sigma\right)
$$

where

$$
A_{1}=\frac{b\left(\frac{1}{B}-\frac{\bar{\gamma}_{\mathrm{mob}}}{B^{2} \varepsilon_{\mathrm{vd}, \mathrm{ir}, \mathrm{ult}}}\right)}{\left(\frac{\bar{\gamma}_{1}^{\mathrm{p}}}{\bar{\gamma}_{\max }}+b\right)^{2}}
$$

Once $\varepsilon_{\mathrm{vd}}$, ir is obtained, the damage variable, $D$, is derived according to Eq. (7).

\section{Reversible Volumetric Strain due to Dilatancy}

$\varepsilon_{\mathrm{vd}, \mathrm{re}}$ is divided into a homotropic reversible dilatancy component, $\varepsilon_{\mathrm{vd}, \mathrm{re}, \mathrm{h}}$, and an aeolotropic component, $\varepsilon_{\mathrm{vd}, \mathrm{re}, \mathrm{a}}$. The dilatancy equation of $\varepsilon_{\mathrm{vd}, \mathrm{re}, \mathrm{h}}$ is assumed as:

$$
\frac{d \varepsilon_{\mathrm{vd}, \mathrm{re}, \mathrm{h}}}{d \bar{\gamma}_{\mathrm{re}, \mathrm{h}}}=\frac{1}{\bar{\mu}_{\mathrm{h}}}\left(M_{0} \mp \frac{|\tau|}{\sigma}\right)
$$

where the operator " - ", is adopted if the interface is loaded and " + " is adopted if the interface is unloaded, which is judged using Eq. (13). $\bar{\gamma}_{\mathrm{re}, \mathrm{h}}$ is the effective shear strain corresponding to the homotropic reversible dilatancy component. $M_{0}$ is the dilatancy angle, which is set to 0.5 at the initial state and decreases to zero at the ultimate state. $\bar{\mu}_{\mathrm{h}}$ is dependent on the normal stress and is used to adjust the $\varepsilon_{\mathrm{vd}, \mathrm{re}, \mathrm{h}} \sim \bar{\gamma}_{\mathrm{re}, \mathrm{h}}$ relationship curve.

$\varepsilon_{\mathrm{vd}, \mathrm{re}, \mathrm{h}}$ at the initial and ultimate states are derived, respectively, by considering the effect of shear strain as expressed in Eq. (20):

$$
\begin{cases}\frac{d \varepsilon_{\mathrm{vd}, \mathrm{re}, \mathrm{h}}}{d \gamma^{\mathrm{p}}}=\frac{1}{\mu_{\mathrm{i}}}\left(M_{0} \mp \frac{|\tau|}{\sigma}\right), & \mu_{\mathrm{i}}=\frac{\mu_{0}}{b_{\mu}}\left(\frac{\sigma}{p_{0}}\right)^{\mathrm{m}_{0}}\left(\frac{\gamma_{1}^{\mathrm{p}}}{\bar{\gamma}_{\max }}+b_{\mu}\right)^{2} \\ \frac{d \varepsilon_{\mathrm{vd}, \mathrm{re}, \mathrm{h}}}{d \gamma^{\mathrm{p}}}=\mp \frac{H_{\mathrm{ru}}}{\mu_{\mathrm{u}}} & \text { at initial state }\end{cases}
$$

where the operator " -" is adopted if the interface is loaded and " + " is adopted if the interface is unloaded, which is judged using Eq. (13). The initial homo-dilatancy modulus, $\mu_{0}$, and homo-dilatancy index, $m_{0}$, are model parameters. $b_{\mu}, n_{\mathrm{b} \mu}$, and $\mu_{\mathrm{u}}$ can be set to constants of 0.15 , 0.5 , and 400, respectively.

The aeolotropic reversible dilatancy component, $\varepsilon_{\mathrm{vd}, \mathrm{re}, \mathrm{a}}$, is dependent on the shear strain and initial loading history. Assume its dilatancy equation to be:

$$
\frac{d \varepsilon_{\mathrm{vd}, \mathrm{re}, \mathrm{a}}}{d \bar{\gamma}_{\mathrm{re}, \mathrm{a}}}=\bar{k}_{\mathrm{a}} I
$$

where $\bar{\gamma}_{\text {re, a }}$ is the effective shear strain corresponding to aeolotropic reversible dilatancy component and $\bar{k}_{\mathrm{a}}$ is dependent on the normal stress and is used to adjust the $\varepsilon_{\mathrm{vd}, \mathrm{re}, \mathrm{a}} \sim \bar{\gamma}_{\mathrm{re}, \mathrm{a}}$ relationship curve. $I$ is introduced to describe the extent of aeolotropy of interface, and has the following attributes: (1) $-1 \leq I \leq 1$; (2) $I=0$ if loading is monotonic; (3) $I$ is a function of the initial stress history if loading is cyclic. Considering a three-dimensional condition, $I$ is simply assumed to be:

$$
I=\left(\frac{\rho}{\rho_{0}}\right)_{\mathrm{i}, \max } \cos \theta^{\prime}
$$

where $\left(\rho / \rho_{0}\right)_{i, \max }$ is the maximum value of $\rho / \rho_{0}$ at the first monotonic loading path. $\theta^{\prime}$ is the angle between the direction of the current shear stress increment and the average direction of the first monotonic loading path. Here such an average direction can be obtained using the direction angle weighted by the magnitude of shear stress increments in such a path.

Assume that $\bar{k}_{\mathrm{a}}$ is an exponential function of the normal stress at the initial state and a constant at the ultimate state. The dilatancy equations for $\varepsilon_{\mathrm{vd}, \text { re, a }}$ at the initial and ultimate states are derived from Eq. (20), as follows:

$$
\begin{cases}\frac{d \varepsilon_{\mathrm{vd}, \mathrm{re}, \mathrm{a}}}{d \gamma^{\mathrm{p}}}=k_{0} I\left(\frac{\sigma}{p_{\mathrm{a}}}\right)^{\mathrm{m}_{\mathrm{k} 0}} \frac{b_{\mathrm{k}}}{\left(\frac{\gamma_{1}^{\mathrm{p}}}{\bar{\gamma}_{\mathrm{max}}}+b_{\mathrm{k}}\right)^{2}} & \text { at initial state } \\ \frac{d \varepsilon_{\mathrm{vd}, \mathrm{re}, \mathrm{a}}}{d \gamma^{\mathrm{p}}}=k_{\mathrm{u}} I \frac{b_{\mathrm{k}}}{\left(\frac{\gamma_{1}^{\mathrm{p}}}{\bar{\gamma}_{\max }}+b_{\mathrm{k}}\right)^{2}} & \text { at utimate state }\end{cases}
$$

where the initial aeolo-dilatancy modulus, $k_{0}$, and aeolo- 
dilatancy index, $m_{\mathrm{k} 0}$, are model parameters. $b_{\mathrm{k}}$, and $k_{\mathrm{u}}$ can be set to constants of 1.5 and 0.04 , respectively.

Using Eq. (6), the homotropic and aeolotropic reversible dilatancy components at an intermediate state are expressed as follows:

$$
\begin{aligned}
& d \varepsilon_{\mathrm{vd}, \mathrm{re}, \mathrm{h}}=\frac{1}{\mu}\left(\frac{1}{H_{\mathrm{r}}} d \tau-\frac{1}{H_{\mathrm{rd}}} \frac{|\tau|}{\sigma} d \sigma\right), \\
& \frac{1}{\mu}=\frac{1-D}{\mu_{\mathrm{i}}}\left(M_{0} \mp \frac{|\tau|}{\sigma}\right) \mp D \frac{H_{\mathrm{r}}}{\mu_{\mathrm{u}}} \\
& d \varepsilon_{\mathrm{vd}, \mathrm{re}, \mathrm{a}}=n_{\mathrm{a}}\left(\frac{1}{H_{\mathrm{r}}} d \tau-\frac{1}{H_{\mathrm{rd}}} \frac{|\tau|}{\sigma} d \sigma\right), \\
& n_{\mathrm{a}}=k I \frac{b_{\mathrm{k}}}{\left(\frac{\gamma_{1}^{\mathrm{p}}}{\bar{\gamma}_{\mathrm{max}}}+b_{\mathrm{k}}\right)^{2}}, \\
& k=(1-D) \cdot k_{0}\left(\frac{\sigma}{p_{\mathrm{a}}}\right)^{\mathrm{m}_{\mathrm{k} 0}}+D \cdot k_{\mathrm{u}}
\end{aligned}
$$

where the operator " - " is adopted if the interface is loaded and " + "' is adopted if the interface is unloaded, which is judged using Eq. (13).

\section{Volumetric Strain due to Compression}

On the basis of test results, we propose a logarithmic relationship between the normal stress and the volumetric strain due to compression. Thus, the differential form of elastic strain is:

$$
d \varepsilon_{\mathrm{vc}}^{\mathrm{e}}=C_{\mathrm{e}} \frac{d \sigma}{\sigma}
$$

where model parameter $C_{\mathrm{e}}$ is the elastic compression index. The bounding surface of compression, $\boldsymbol{F}_{\mathrm{v}}$, is expressed as:

$$
\boldsymbol{F}_{\mathrm{v}}=\sigma-\infty=0
$$

where $\infty$ represents infinite. The plastic volumetric strain due to compression is assumed to be zero at the ultimate state. Therefore, according to the decomposition of plastic strain assumption, the plastic volumetric strain due to compression is derived as:

$$
d \varepsilon_{\mathrm{vc}}^{\mathrm{p}}=\frac{C}{\sigma} d \sigma, \quad \begin{cases}C=C_{0}(1-D), & \sigma+d \sigma>\sigma_{\max } \\ C=0, & \sigma+d \sigma \leq \sigma_{\max }\end{cases}
$$

where the model parameter $C_{0}$ is the plastic compression index. $\sigma_{\max }$ is the maximum historic normal stress that the interface has undergone.

\section{Mathematical Formulation}

Mathematical formulations of the EPDI model are derived according to Eq. (8) and the above formulae of strain components, that is,

$$
\left\{\begin{array}{l}
d \gamma=\left(\frac{1}{G_{\mathrm{e}}}+\frac{1}{H_{\mathrm{r}}}\right) d \tau-\frac{1}{H_{\mathrm{rd}}} \frac{|\tau|}{\sigma} d \sigma \\
d \varepsilon_{\mathrm{v}}=\frac{\frac{1}{\mu}+n_{\mathrm{a}}+A_{1}}{H_{\mathrm{r}}} d \tau+\left(\frac{C+C_{\mathrm{e}}}{\sigma}-\frac{\frac{1}{\mu}+n_{\mathrm{a}}+A_{1}}{H_{\mathrm{rd}}} \frac{|\tau|}{\sigma}\right) d \sigma
\end{array}\right.
$$

The expressions of the variables in the equation can be found in the corresponding strain components from Eqs. (16), (22), (28), and (29).

\section{PARAMETERS}

A total of twelve parameters are required to be determined for the EPDI model. The parameters are meaningful for physical concepts and divided into six groups (Table 1). Only a certain number of parameters are needed to describe a particular aspect of the behavior of the interface; this demonstrates that the number of parameters may be furthermore reduced. For example, only ten parameters are needed if aeolotropy of interface is insignificant.

All the parameters are determined using a group of cyclic shear tests under constant normal stress condition and a confining compression test. To determine the parameters, we recommend the following steps:

(1) A group of cyclic shear tests of the interface are conducted under constant normal stress condition. The displacements are converted to strain according to the thickness of the interface. It should be noted that this thickness can be determined by measuring the movements of soil particles. The thickness can be also estimated as five times the average grain-size of soil for a common interface.

(2) Determination of damage parameters. $\varepsilon_{\mathrm{vd}}$, ir is separated from the volumetric strain due to dilatancy by obtaining its maximum value within each shear cycle (referred to Fig. 2). Thus, the parameters $\varepsilon_{\mathrm{vd}}$, ir, ult, $\alpha$, and $\beta$ are determined by fitting the relationship between $\varepsilon_{\mathrm{vd}}$, ir and number of shear cycles using Eq. (18) ( $\bar{\gamma}$ is set to 2 for a single shear cycle).

(3) Determination of strength parameters. The friction angle, $\varphi_{0}$, is obtained by fitting the relationship between the shear strength and normal stress using Eq. (9).

(4) Determination of shear strain parameters. The parameters $G_{0}$ and $n_{0}$ are obtained by fitting the monotonic shear stress-strain relationship using Eq. (33).

(5) Determination of parameters of reversible dilatancy component. The parameters $\mu_{0}$ and $m_{0}$ are determined by fitting the monotonic relationship between volumetric strain and shear strain with reference to the obtained $\varepsilon_{\mathrm{vd}}$, ir using Eq. (28).

(6) Determination of aeolotropy parameters. The parameters $k_{0}$ and $m_{\mathrm{k} 0}$ are determined by fitting the relationship between volumetric strain and shear strain due to the application of reverse shear with reference to the obtained $\varepsilon_{\mathrm{vd}}$, ir and $\varepsilon_{\mathrm{vd}, \text { re, h }}$ using Eq. (29). 
Table 1. Parameters of the EPDI model and their values for two types of interfaces

\begin{tabular}{|c|c|c|c|c|}
\hline \multirow{2}{*}{ Group } & \multirow{2}{*}{ Parameters } & \multirow{2}{*}{ Symbol } & \multicolumn{2}{|c|}{ Value } \\
\hline & & & $1 \#$ & $2 \#$ \\
\hline \multirow{3}{*}{ Damage } & $\begin{array}{l}\text { maximum irreversible } \\
\text { volumetric strain due to } \\
\text { dilatancy }\end{array}$ & $\varepsilon_{\mathrm{vd}, \text { ir , ult }}$ & 0.35 & 0.24 \\
\hline & $\begin{array}{l}\text { irreversible dilatancy } \\
\text { modulus }\end{array}$ & $\alpha$ & 250 & 95 \\
\hline & $\begin{array}{l}\text { irreversible dilatancy } \\
\text { index }\end{array}$ & $\beta$ & 1.18 & 0.5 \\
\hline Strength & friction angle & $\varphi\left({ }^{\circ}\right)$ & 38 & 42.5 \\
\hline \multirow{2}{*}{ Shear strain } & initial shear modulus & $G_{0}$ & 100 & 105 \\
\hline & initial shear index & $n_{0}$ & 0.32 & 0.69 \\
\hline \multirow{2}{*}{$\begin{array}{l}\text { Reversible } \\
\text { dilatancy } \\
\text { component }\end{array}$} & $\begin{array}{l}\text { initial homo-dilatancy } \\
\text { modulus }\end{array}$ & $\mu_{0}$ & 6 & 39 \\
\hline & homo-dilatancy index & $m_{0}$ & 0.8 & 0.31 \\
\hline \multirow[t]{2}{*}{ Aeolotropy } & $\begin{array}{l}\text { initial aeolo-dilatancy } \\
\text { modulus }\end{array}$ & $k_{0}$ & 0.14 & 0.02 \\
\hline & aeolo-dilatancy index & $m_{\mathrm{k} 0}$ & 0.7 & -0.1 \\
\hline \multirow{2}{*}{ Compression } & elastic compression index & $C_{\mathrm{e}}$ & 0.005 & 0.005 \\
\hline & initial compression index & $C_{0}$ & 0.005 & 0.005 \\
\hline
\end{tabular}

$1 \#$ : steel-gravel interface (thickness: $50 \mathrm{~mm}$ )

2\#: concrete-gravel interface of Zipingpu concrete-faced rockfill dam (thickness: $100 \mathrm{~mm}$ )

(7) Determination of compression parameters. A confining compression test, including loading and unloading, is used to determine the parameters $C_{\mathrm{e}}$ and $C_{0}$ by fitting the relationship between volumetric strain and normal stress using Eqs. (30) and (31).

\section{VERIFICATION}

To verify the effectiveness of the EPDI model, we compared model predictions of the monotonic and cyclic responses of the interfaces between structures and gravels with serialized test results. The test method has been described in a previous paper (Zhang and Zhang, 2006b). Two types of interface are used in this paper for the verification and their model parameters are listed in Table 1. One is the steel-gravel interface that is between a steel plate (roughness degree is $1 \mathrm{~mm}$ ) and homo-gravel (Fig. 10(a), Figs. 11-13) that has been described previously (Zhang and Zhang, 2006b). The other is the concretegravel interface between a concrete slab and gravel of the 156m-high Zipingpu concrete-faced rockfill dam (CFRD) (Fig. 10(b), Fig. 14); the average grain size of the gravel is approximately $20 \mathrm{~mm}$ and the designed dry density is 2.15 $\mathrm{g} / \mathrm{cm}^{3}$.

Figure 10 shows a comparison of test results and model predictions of the monotonic stress-strain relationship for two types of interfaces under constant normal stress boundary condition. The predicted curve shows a good fit to the test result. This demonstrates that the EPDI model provides a reasonable characterization of the monotonic stress-strain relationship, including shear behavior and volumetric change (e.g., dilation if normal stress is small;
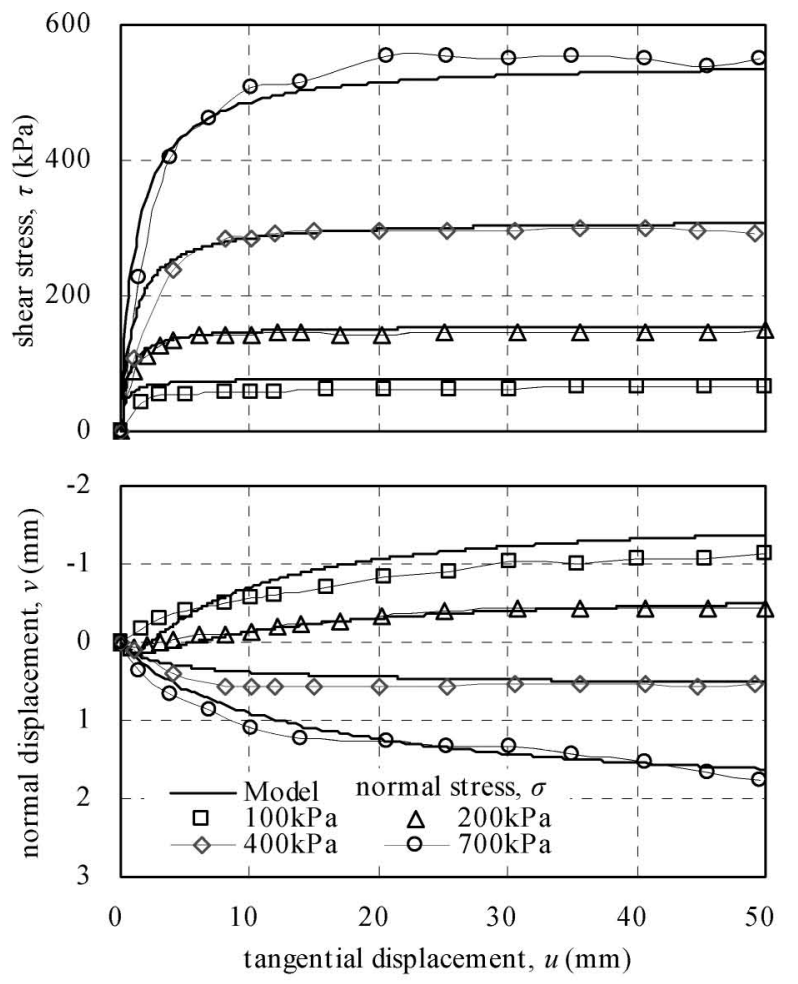

(a)
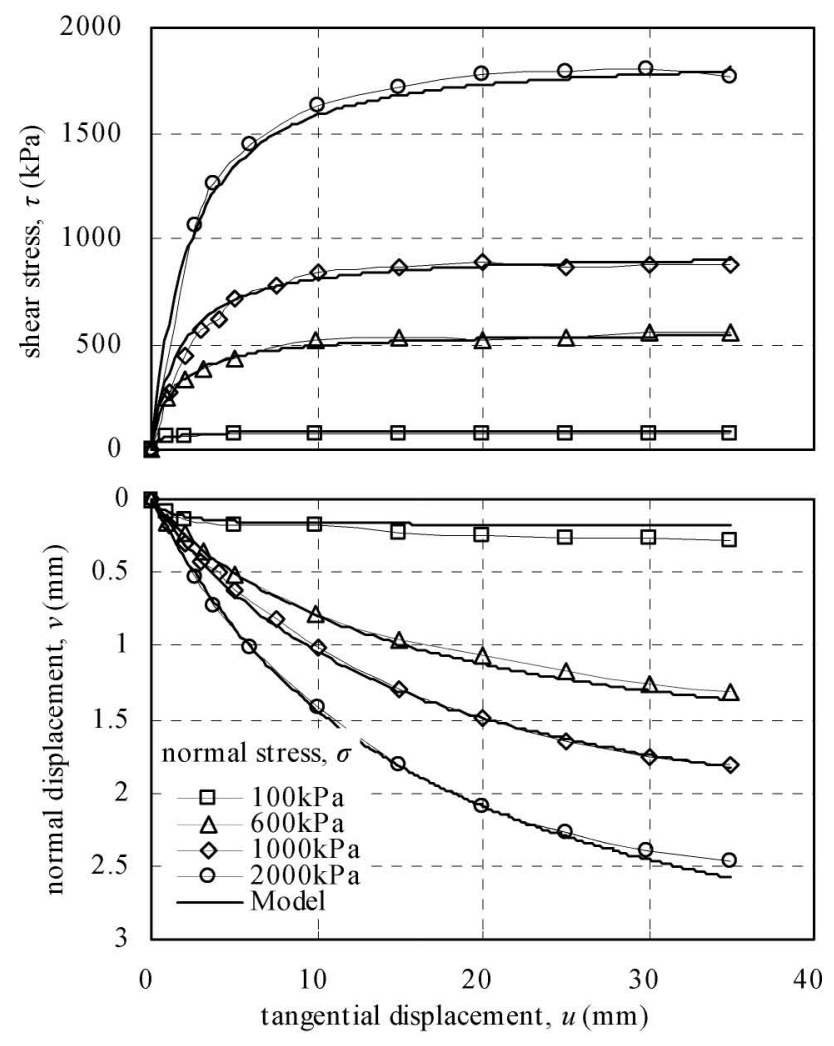

(b)

Fig. 10. Comparison of model predictions and test results of monotonic stress-displacement relationship of an interface under constant normal stress condition: (a) steel-gravel interface and (b) concrete-gravel interface of Zipingpu CFRD symbol: test result; line: model prediction 

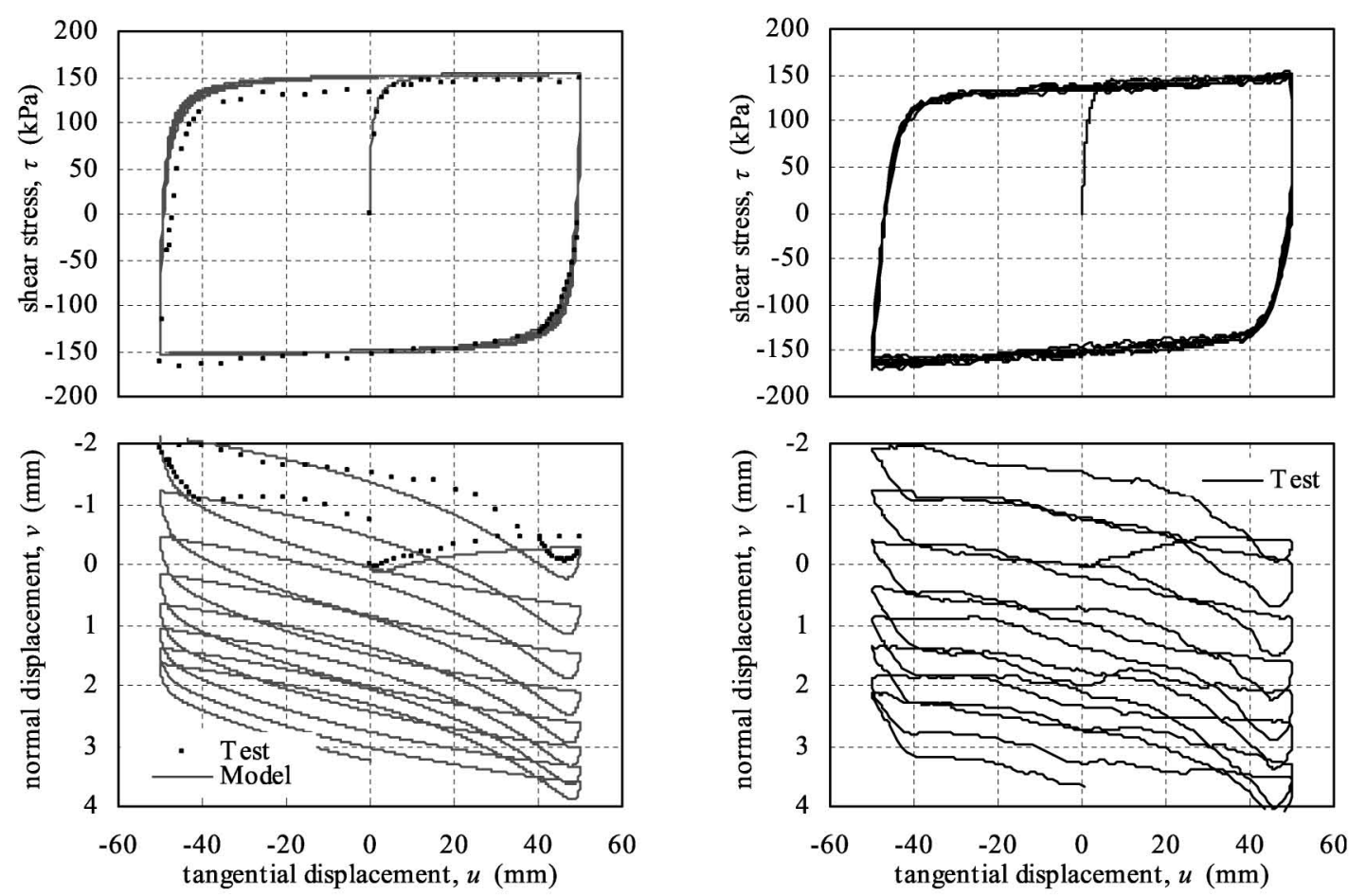

(a)
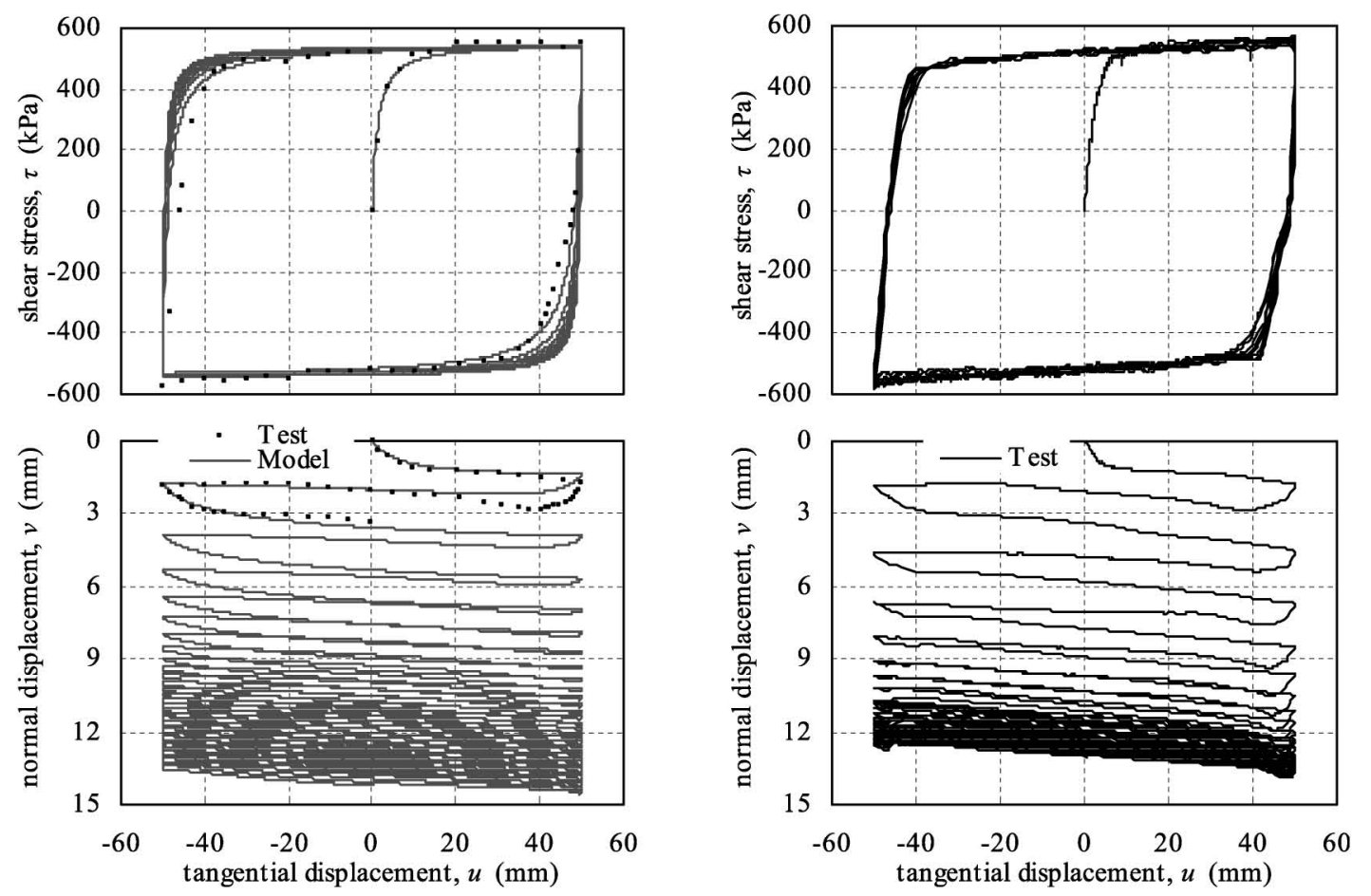

(b)

Fig. 11. Comparison of model predictions and test results of cyclic stress-displacement relationship of steel-gravel interface under constant normal stress condition: (a) normal stress: $200 \mathrm{kPa}$ and (b) normal stress: $700 \mathrm{kPa}$ 

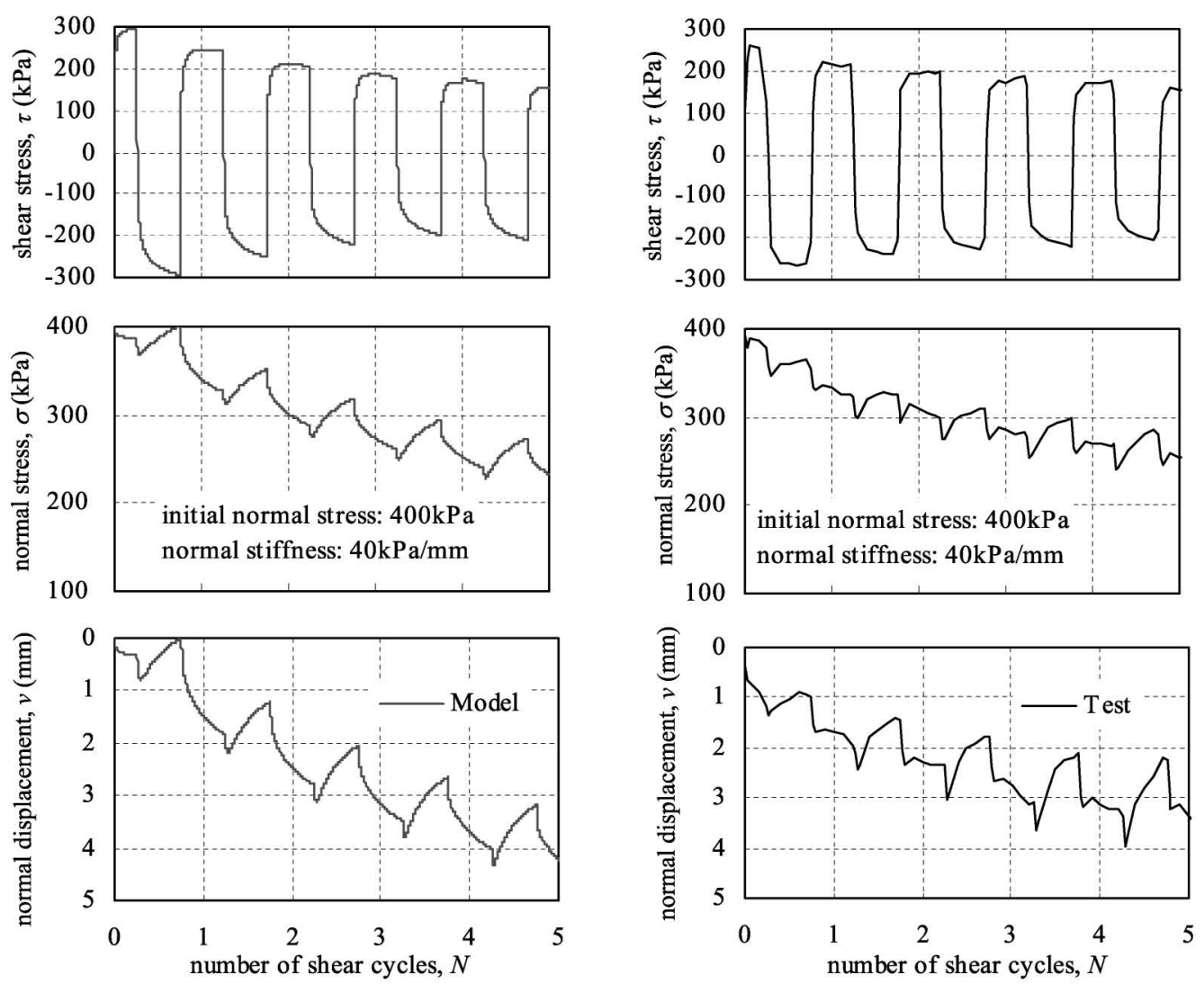

(a)
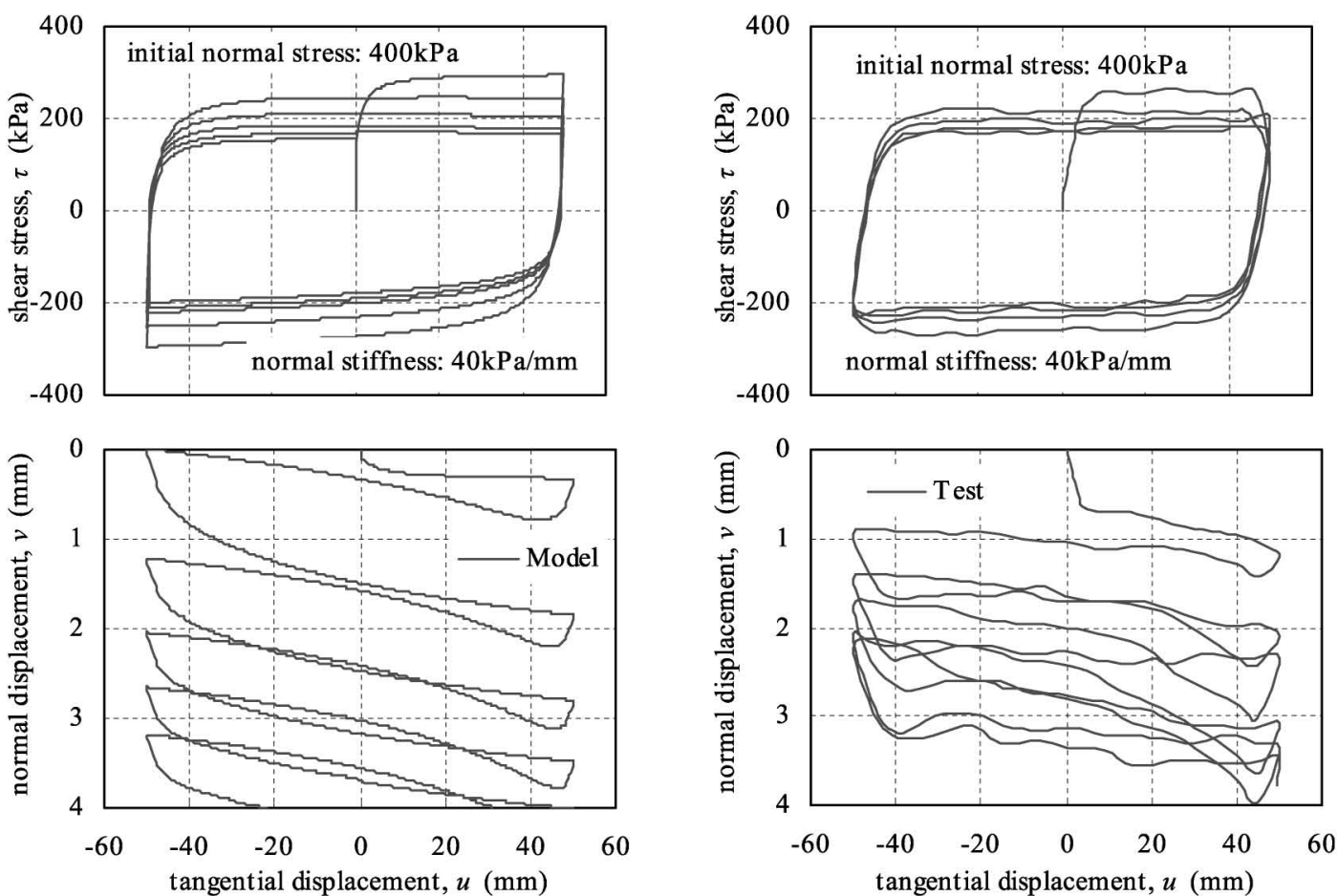

(b)

Fig. 12. Comparison of model predictions and test results of cyclic response of steel-gravel interface under constant normal stiffness: (a) history and (b) stress-displacement relationship 

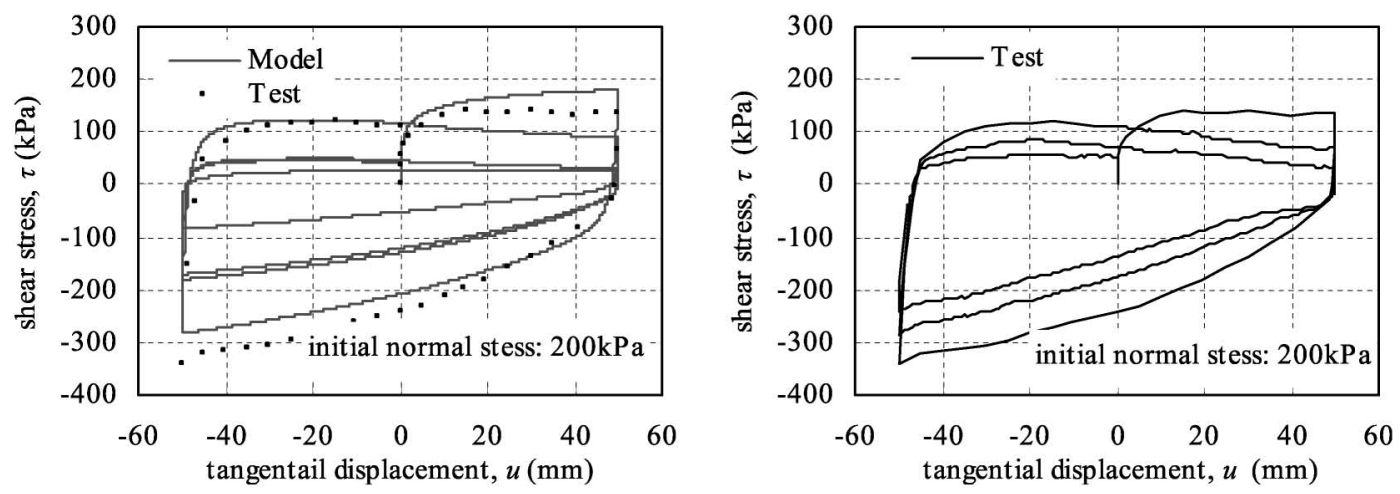

Fig. 13. Comparison of model predictions and test results of cyclic stress-displacement relationship of steel-gravel interface under constant normal displacement condition

contraction if normal stress is large).

Figures 11-13 provide a comparison of test results and model predictions of the cyclic response of the steel-gravel interface under various normal boundary conditions, including constant stress, constant stiffness and constant displacement. Figure 14 compares the test results and model predictions of the cyclic stress-strain relationship of the concrete-gravel interface under constant normal stress boundary condition. A few test results are also plotted alongside the model predictions to enable a direct comparison of predicted and observed behavior. For the constant normal stiffness boundary condition, the following equation was substituted to the Eq. (32) to derive the stress-strain relationship of the interface.

$$
\frac{d \sigma}{d \varepsilon_{\mathrm{v}}}=-K
$$

where $K$ is the normal stiffness used in the test. It should be noted that the model parameters are determined using the test results under constant normal stress boundary condition; thus the model predictions on the response of the interface under other normal boundary conditions, including constant stiffness and constant displacement, are independent from the model parameters. The test results under multiple normal boundary conditions provides an effective approach to confirm the effectiveness of the model. These comparisons demonstrate that the model prediction curves are in good agreement with the test results under multiple kinds of cyclic loading conditions and different interfaces. Therefore, we conclude that the EPDI model is effective in capturing the primary cyclic behaviors of the interface, including volumetric change due to dilatancy, aeolotropy of interface, compression due to unloading, and shear stress-strain relationship response with the change of normal stress.

\section{CONCLUSIONS AND REMARKS}

(1) We present some new concepts with a solid testbasis, including: 1) Volumetric strain due to dilatancy consists of reversible and irreversible dilatancy components. 2) Aeolotropy of interface is used to describe the feature that mechanical response, especially volumetric change, is dependent on the shear direction. 3) Effective shear strain is used to consider the feature that volumetric strain due to dilatancy is induced mainly by the deformation of the soil constrained by the structure that is a part of tangential displacement. 4) Significant evolution of the physical state is induced by shear application and results in evolution of behavior from the initial state and to a stable state; the evolutions can be measured using the irreversible volumetric strain due to dilatancy.

(2) We propose a model framework that is designed for an interface between a structure and gravelly soil based on existing model theory and our understanding of deformation mechanism of the interface. The framework has the following attributes: 1) The framework simplifies the evolution of behavior to that of certain behavior parameters. 2) The framework respectively formulates the two plastic strain components due to shear and compression using a division of plastic strain assumption. 3) The framework uses a bounding surface scheme to compute the plastic shear strain due to shear application. 4) The framework directly presents the dilatancy equation used to compute the volumetric strain due to dilatancy.

(3) We present a new elasto-plasticity damage model, the EPDI model, established on a solid test basis. The parameters are easily determined from a group of cyclic shear tests under constant normal stress condition and a confining compression test.

(4) We demonstrate the effectiveness of the EPDI model by comparing model predictions with the results for a series of tests under varying loading conditions. The model accurately describes monotonic and cyclic stressstrain relationship of the interface between a structure and gravelly soil with successfully capturing new features, including: 1) shear stress-strain relationship and comprehensive volumetric strain response (e.g., partly dependent on shear strain), especially under cyclic loading condition; 2) volumetric strain response that is dependent on the shear direction; and 3) evolution of behavior of the interface associated with the evolution of physical state. 

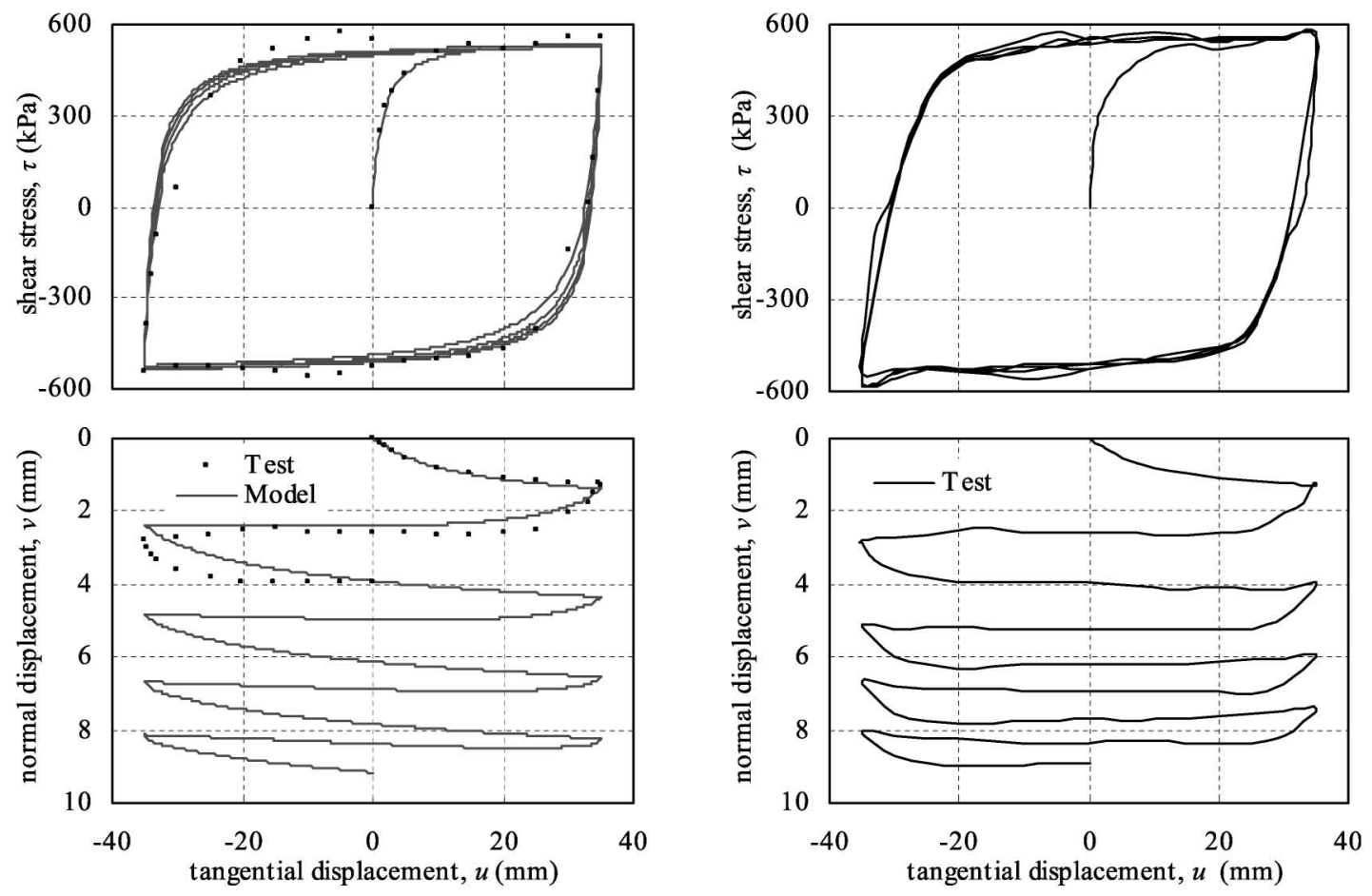

(a)
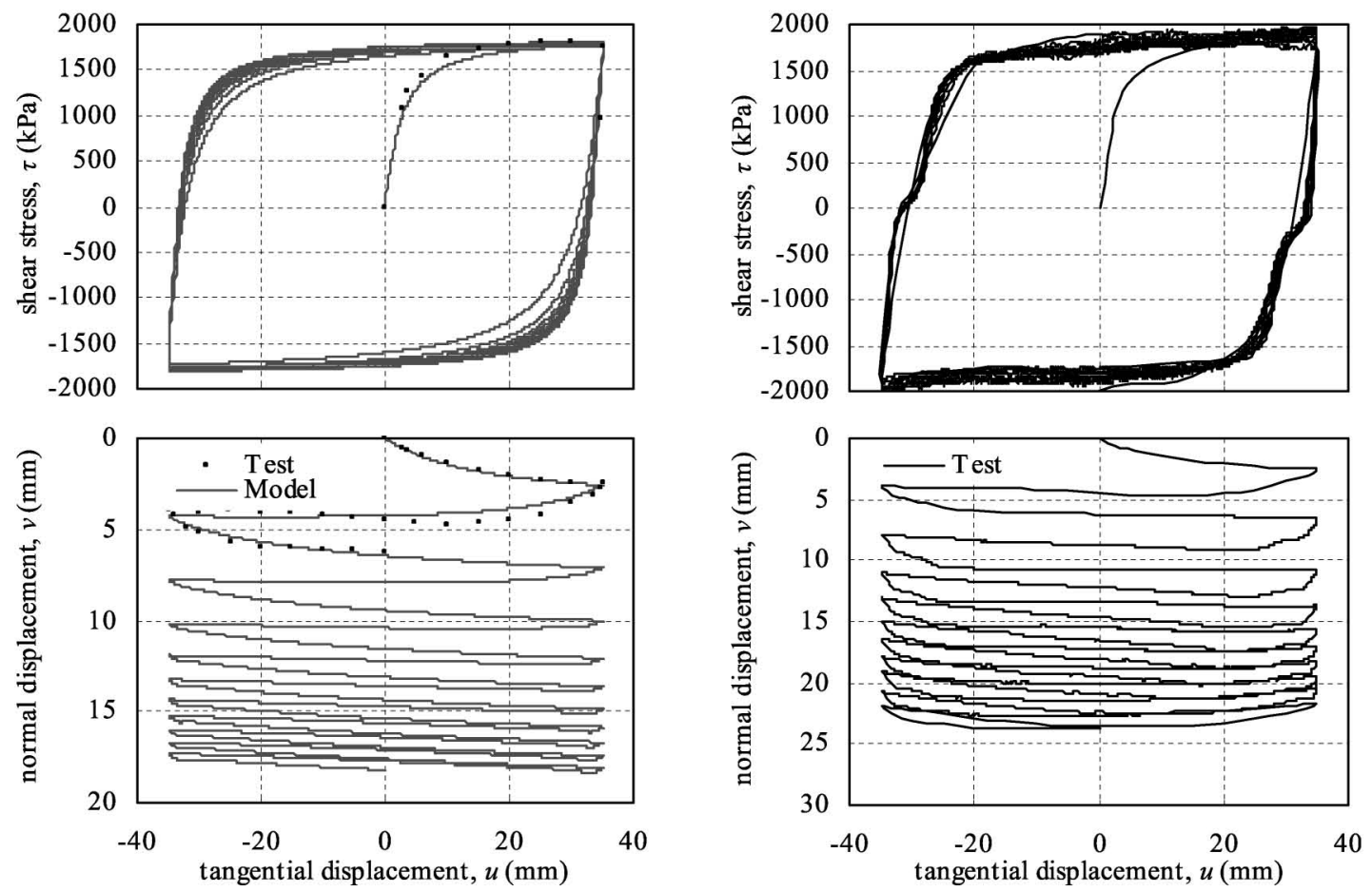

(b)

Fig. 14. Comparison of model predictions and test results of cyclic response of concrete-gravel interface of Zipingpu CFRD under constant normal stress condition: (a) normal stress: $600 \mathrm{kPa}$ and (b) normal stress: $2000 \mathrm{kPa}$

\section{ACKNOWLEDGEMENTS}

The project is supported by National Basic Research Program of China (973 Program) (No. 2007CB714108) and National Natural Science Foundation of China (No.
50679034).

\section{REFERENCES}

1) Boulon, M. and Nova, R. (1990): Modeling of soil-structure inter- 
face behavior: a comparison between elastoplastic and rate type laws, Computers \& Geotechnics, (9), 21-46.

2) Brandt, J. R. T. (1985): Behavior of soil-concrete interfaces, Ph.D Thesis, Edmonton, Alberta, Canada, University of Alberta.

3) Clough, G. W. and Duncan, J. M. (1971): Finite element analysis of retaining wall behavior, J Soil Mech. \& Found. Div. ASCE, 97 (SM12), 1657-1672.

4) Dafalias, Y. F. and Herrmann, L. R. (1980): A bounding surface soil plasticity model, Soils under Cyclic and Transient Loading, 1, 335-346.

5) Desai, C. S., Drumm, E. C. and Zaman, M. (1985): Cyclic testing and modeling of interfaces, $J$ Geotech. Div., ASCE, 111(6), 793-815.

6) Desai, C. S. and Ma, Y. (1992): Modeling of joints and interfaces using the disturbed-state concept, Int. J Numerical \& Analytical Method in Geomechanics, 16, 623-653.

7) Fakharian, K. and Evgin, E. (2000): Elasto-plastic modeling of stress-path-dependent behavior of interfaces, Int. J Nume. \& Analy. Method in Geomechanics, 24, 183-199.

8) Fishman, K. L. and Desai, C. S. (1987): A constitutive model for hardening behavior of rock joints, Desai et al., Editors, Constitutive Laws for Engineering Materials: Theory and Applications, 1043-1050.

9) Fishman, K. L., Derby, C. W. and Plamer, M. C. (1991): Verification for numerical modeling of jointed rock mass using thin layer elements, Int. J Nume. \& Analy. Method in Geomechanics, 15, 61-70.

10) Frantziskonis, G. and Desai, C. S. (1987): Elasto-plastic model with damage for strain softening geomaterials, Acta Mechanica, 68, 151-170.

11) Gens, A., Carol, I. and Alonso, E. E. (1990): A constitutive model for rock joints formulation and numerical implementation, Com- puters \& Geotechnics, (9), 3-20.

12) Ghaboussi, J., Wilson, E. L. and Isenberg, J. (1973): Finite element for rock joints and interfaces, J. Soil Mech. Found. Div., ASCE, 99(SM10), 833-848.

13) $\mathrm{Hu}, \mathrm{L}$. and $\mathrm{Pu}, \mathrm{J}$. (2004): Testing and modeling of soil-structure interface, J Geotech. \& Geoenv. Eng., ASCE, 130(8), 851-860.

14) Kachanov, L. M. (1958): On the time to failure under creep condition, Izv. Akad. Nauk SSSR, Otd. Tekh. Nauk, Metall. Topl., 8, 26-31.

15) Mroz, Z. (1967): On the description of anisotropic hardening, $J$ Mech. \& Physics of Solids, 15, 163-175.

16) Navayogarajah, N., Desai, C. S. and Kiousis, P. D. (1992): Hierarchical single-surface model for static and cyclic behavior of interfaces., J Eng. Mech., ASCE, 118(5), 990-1011.

17) Pal, S. and Wathugala, G. W. (1999): Disturbed state model for sand-geosynthetic interfaces and application to pull-out tests, Int. $J$ Nume. \& Analy. Method in Geomechanics, 23, 1873-1892.

18) Shen, Z. J. and Zhang, W. M. (1988): Application of damage in soil mechanics, Proc. 3rd National Nume. \& Anal. Methods in Geomechanics, 1-8 (in Chinese).

19) Uesugi, M. and Kishida, H. (1986): Frictional resistance at yield between dry sand and mild steel, Soils and Foundations, 26(4), 139-149.

20) Zhang, G. and Zhang, J.-M. (2006a): A large-scale apparatus for monotonic and cyclic soil-structure interface test, Geotechnical Testing Journal, 29(5), 401-408.

21) Zhang, G. and Zhang, J.-M. (2006b): Monotonic and cyclic tests of interface between structure and gravelly soil, Soils and Foundations, 46(4), 505-518.

22) Zhang, G., Liang, D. and Zhang, J.-M. (2006): Image analysis measurement of soil particle movement during a soil-structure interface test, Computers and Geotechnics, 33(4-5), 248-259. 